\title{
FEM simulation of soft tissues
}

\author{
Z. Zhou \& D. M. Bruce \\ Division of Bio-Engineering \\ Silsoe Research Institute, UK
}

\begin{abstract}
This paper is to present a model simulating the mechanical behaviour of soft tissues based on large displacement theory. In this model, large deformation characteristic of soft tissues is described with an incremental non-linear geometric equation. Galerkin weighted residual method is used to produce finite element formulations. Computer program is written to implement the FEM solution. A software package is thus created for simulation of the mechanical behaviour of soft tissues in situations such as surgery and wound care. The numerical model was applied to the simulation of a hypothetical example of wound suturing. Numerical results from the analysis are presented and discussed.
\end{abstract}

\section{Introduction}

Over last fifteen years, the finite element method (FEM) has increasingly been adopted as an approach to the analysis of soft tissue [1] and has found application in various areas. Much research has been carried out using FEM without including geometric non-linearity [2][3][4][5][6][7]. These works have indicated that the finite element method can be a powerful tool for soft tissue simulation.

One of the typical mechanical behaviours of soft tissues is large deformation even under normal working conditions ([8]). Geometric non-linearity should be considered in soft tissue modelling in order to take account of the large deformation behaviour. Lott-Crumpler and Chaudhry [9] have applied the finite element method to investigate the optimal patterns for suturing wounds of complex shapes to foster healing. In their investigation, non-linearity of the geometric equation was included. A detailed description of soft tissue simulation including geometric nonlinearity was presented by Maurel et al [10]. 


\section{4}

Simulations in Biomedicine $V$

The approach to derive the finite element formulation adopted by Maurel et al [10] was based on the virtual work principle. A number of studies in the area of classical nonlinear mechanics have been carried out on finite element analysis considering geometric non-linearity based on the virtual work principle [11] $[12][13][14]$.

With the virtual work principle approach, it is not easy to address the problem of coupled mechanical and hydraulic behaviour of media. A study has been carried out to derive the finite element formulation including the geometric non-linearity based on Galerkin weighted residual method [15][16]. This approach will here be further developed for the simulation of the mechanical behaviour of biological soft tissues.

In this paper, a numerical approach considering the geometric non-linearity is presented for the simulation of biological soft tissues. Geometric non-linearity is considered through an incremental approach. Nonlinear finite element formulation is derived based on the Galerkin weighted residual method. The numerical model is then applied to the simulation of a hypothetical example of wound suturing.

\section{Theory}

In the traditional area of mechanics, the displacement, the deformation or the strain of materials such as steel or concrete are required to be 'small' under working load, otherwise mechanical failure may develop or the engineering products may not work properly. In these circumstances, small strain assumption can be used in the theoretical description of the mechanics of such materials and the theory of elasticity can produce very accurate results.

However, materials like soft tissues, such as skin are highly deformable. The high deformability allows soft tissues to stretch and turn in large magnitude that is required by organisms under normal working conditions. The small strain assumption in this case is not appropriate. To describe the large deformation needs to consider the geometric non-linearity. The theoretical description of the large deformation theory, namely, nonlinear mechanics can be found in a number of books (Basar and Weichert, 2000).

In the following sections, an approach is presented to produce a finite element formulation based on the established nonlinear mechanics.

\subsection{Geometric non-linearity}

One of the strain measures describing large displacement is Green-Lagrange strain which is defined in the initial Cartesian coordinate system. The Green-Lagrange strain tensor can be expressed as

$$
E_{i j}=\frac{1}{2}\left(\frac{\partial u_{i}}{\partial X_{j}}+\frac{\partial u_{j}}{\partial X_{i}}+\frac{\partial u_{k}}{\partial X_{i}} \frac{\partial u_{k}}{\partial X_{j}}\right)
$$


where $\mathrm{u}$ is displacement vector, $\mathrm{X}$ is coordinates and $\mathrm{i}$ and $\mathrm{j}$ are subscript indices ( 1 , 2 and 3 ). In small deformation theory, the third term on the right side of equation (1) which is nonlinear is ignored.

The incremental strain can be expressed as

$$
\Delta \mathrm{E}=\frac{1}{2}\left(\frac{\partial \Delta \mathrm{u}_{\mathrm{i}}}{\partial \mathrm{X}_{\mathrm{j}}}+\frac{\partial \Delta \mathrm{u}_{\mathrm{j}}}{\partial \mathrm{X}_{\mathrm{i}}}\right)+\frac{1}{2}\left(\frac{\partial \Delta \mathrm{u}_{\mathrm{k}}}{\partial \mathrm{X}_{\mathrm{i}}} \frac{\partial \mathrm{u}_{\mathrm{k}}}{\partial \mathrm{X}_{\mathrm{j}}}+\frac{\partial \mathrm{u}_{\mathrm{k}}}{\partial \mathrm{X}_{\mathrm{i}}} \frac{\partial \Delta \mathrm{u}_{\mathrm{k}}}{\partial \mathrm{X}_{\mathrm{j}}}\right)
$$

The incremental strain $\Delta \mathrm{E}$ is linear with respect to the incremental displacement $\Delta \mathrm{u}$. This is one of the advantage of incremental model.

\subsection{Stresses and incremental stresses}

In nonlinear mechanics, definitions of three stress tensors are in general use, viz. the Cauchy stress tensor $\sigma$, the Piola-Kirchoff first stress tensor P and the PiolaKirchoff second stress tensors $\mathrm{S}$.

The relationship between Cauchy stress tensor $\sigma$ and the Piola-Kirchoff second stress tensor $\mathrm{P}$ is described by

$\mathrm{S}=\mathrm{JF}^{-1} \sigma\left(\mathrm{F}^{-1}\right)^{\mathrm{T}}$

where $J$ is the determinant of the gradient tensor $F$ and the superscript $T$ is indicates transposition of the matrix.

The relationship between the Piola-Kirchoff first stress tensor and Cauchy stress tensor is

$$
\mathrm{P}=\mathrm{JF}^{-1} \sigma
$$

The relationship between the Piola-Kirchoff second stress tensor and the PiolaKirchoff first stress tensor is

$\mathrm{S}=\mathrm{P}\left(\mathrm{F}^{-1}\right)^{\mathrm{T}}$

In this paper, the incremental form of the Piola-Kirchoff second stress tensor, $\Delta S$ is used as the basic variable. The incremental form of the Piola-Kirchoff second stress tensor is also symmetric. The total Piola-Kirchoff second stress can be obtained by adding together all the previous increments of it. Cauchy stress does not have such additive relationship. 
Although Green -Lagrange strain and Piola-Kirchoff second stress are defined in terms of initial coordinates, they actually follow the material coordinate system. So the directions of the Green -Lagrange strain and the Piola-Kirchoff second stress vary, as does the material coodinate system. It is apparent that the Cauchy stress tensor defined in the current Cartesian coordinate system, is a better choice for displaying results.

\subsection{Equilibrium equations}

Normally, in the virtual work approach, equilibrium equations of forces are not prerequisite but are arise from the virtual work equation. In the approach adopted here, the equilibrium equations are needed. These equations together with the geometric equations and constitutive equations will lead to the establishment of the required governing equations.

The equilibrium equation of forces defined with respect to the initial coordinate system is

$$
\frac{\partial}{\partial X}(P)+f=0
$$

where $\mathrm{f}$ is the body force.

Differentiating both sides of the equation (6) yields

$$
\frac{\partial}{\partial \mathrm{X}}(\Delta(\mathrm{F}) \mathrm{S}+\mathrm{F} \Delta(\mathrm{S}))+\Delta \mathrm{f}=0
$$

Equation system (7) are the incremental equilibrium equations.

\subsection{Constitutive relationship}

The general form of the three-dimensional incremental constitutive relationship can expressed as

$$
\Delta \mathrm{S}=\mathrm{D} \Delta \mathrm{E}
$$

\subsection{Numerical solution and computer code}

It is very difficult to obtain an analytical solution from the model, so a numerical method, in this case, the finite element method is employed to derive an approximate solution. Numerical discretisation can be achieved by applying 
Galerkin's weighted residual method. The numerical solution was then implemented in a computer code BAFEM written with FORTRAN 90.

\section{Application}

To illustrate capabilities of the model, a 2D analysis of a partly sutured "wound" is presented.

\subsection{Problem descriptions}

The analyzed domain is $10 \mathrm{~cm}$ long and $2 \mathrm{~cm}$ thick with a V-shape "wound" located at its middle as shown in figure 1 . The mesh generated using quadrilateral elements is illustrated also in figure 1 .

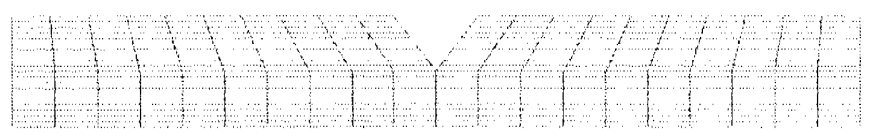

Figure 1 Analysed domain and mesh

A fixed displacement boundary condition was applied at the bottom boundary in both directions. Displacements at the side boundaries were fixed in the $\mathrm{X}$ direction and not constrained in the $\mathrm{Y}$ direction. Suturing of $\mathrm{V}$-shape wound was implemented by "stitching" together the nodes on each side of the wound, so that ten stitches were required to complete the suturing of the whole wound. In this simulation the lower seven stitches have been inserted. Initial stress and strain were assumed to be zero.

Incremental Hooke's law was assumed as the constitutive relationship for soft tissue. According to Fung (1993), the Young's modulus of some skin tissues is around $1 \mathrm{Mpa}$, so this value was used. No data has been found for the Poisson's ratio, so a Poisson's ratio of 0.4 was assumed here.

\subsection{Results and discussions}

The results of displacements is plotted against the deformed tissue domain in figures 2,3 and 4 .

The contours of displacement in X direction after seven stitches are completed are demonstrated in figure 2 . The displacement in the $\mathrm{X}$ direction is anti-symmetric, because wound suturing causes the soft tissue at the two sides of the wound to be 


\section{Simulations in Biomedicine $V$}

pulled towards the centre line. The tissues at the left side of the wound are pulled towards right and vice versa. The largest displacements occur near the suturing sites, which is physical sensible.

The contours of displacement in the y direction after insertion of seven stitches are illustrated in figure 3. Displacements within the blue to orange colour range are negative, i.e., the soft tissues in these zones were pulled downwards. The displacements in the dark red colour zone are positive, meaning that soft tissues in these zones are squeezed upwards. This is the reason that an arch-curved skin surface is observed from the simulation. It is interesting to see that suturing can result in such a complex displacement pattem even in only 2D.

The contours of the total displacement of the displacement vector are displayed in figure 4. Because the displacement vector has two components, one in the $X$ direction and one in the $Y$ direction, figure 4 is actually the synthesis of figure 2 and figure 3. It can be seen that the largest displacements occur at the places near the suturing site. The more remote is the location from the suturing site, the smaller is the displacement.

The vector of the first principal Cauchy stress is displayed in figure 5 . The length of the vector lines indicates the magnitude of the stress. The direction of the vector lines is the direction of the stress. Understandably, the largest stresses occurred at the places of the suturing site. It can be seen that the magnitude of the stresses along the suturing line varied considerably. The stresses at the lower stitching sites are very small compared with the stresses at the upper stitching sites. The first principal stresses in the area below the suturing site are the smallest.
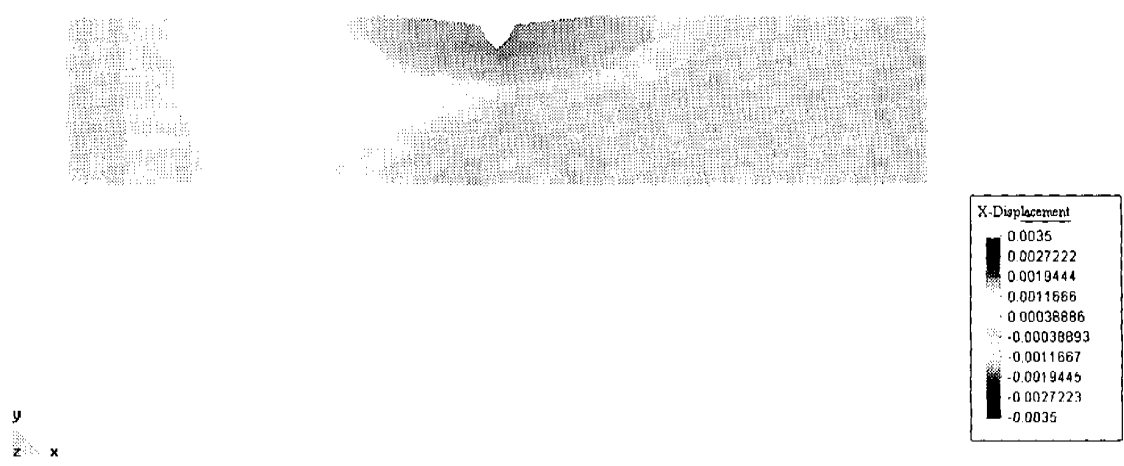

Figure 2: Contour of displacement in $\mathrm{X}$ direction 


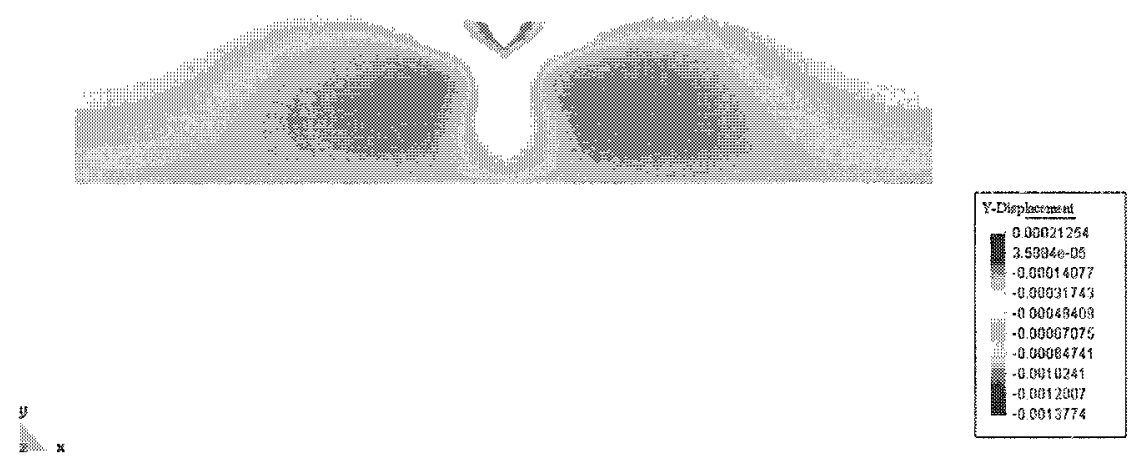

Figure 3: Contour of displacement in $Y$ direction

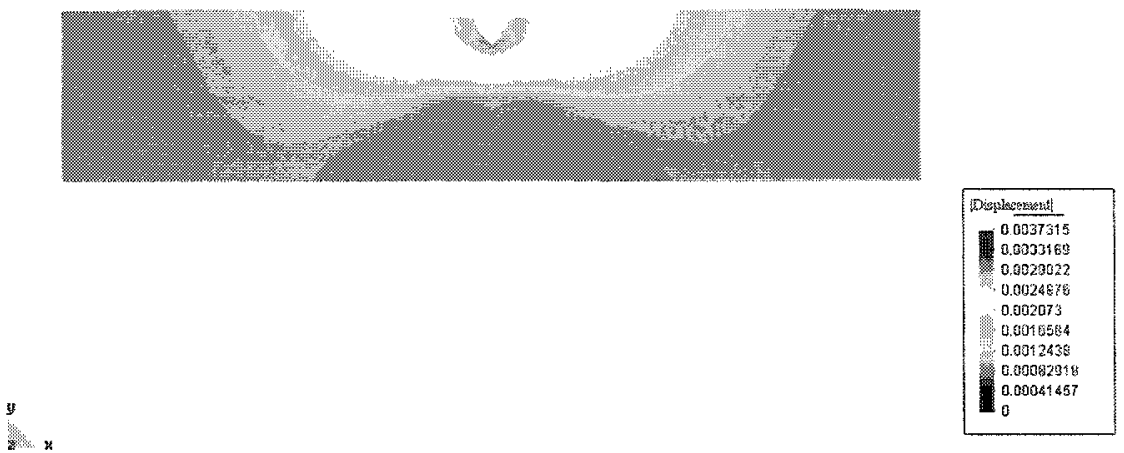

Figure 4: Contour of total displacement 


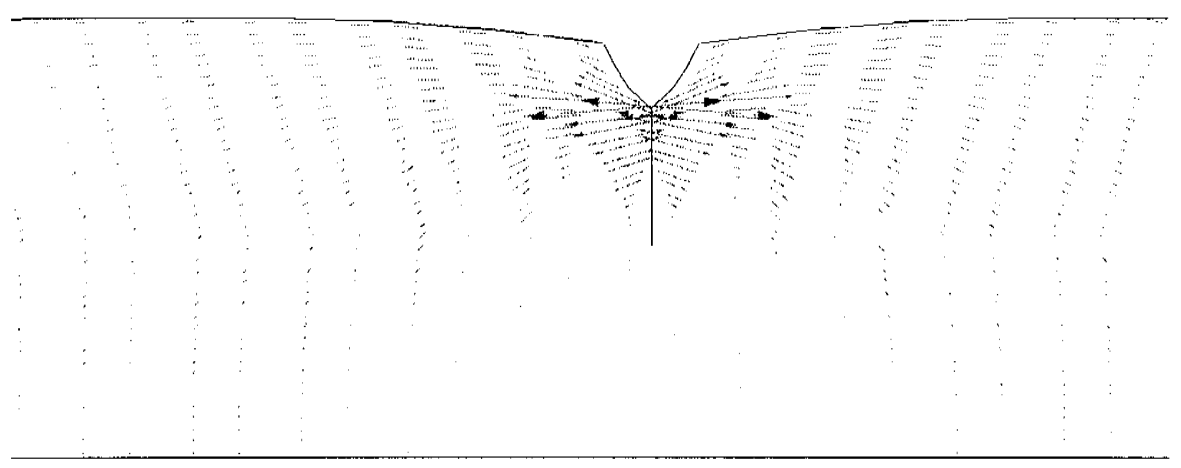

y.

Figure 5 Vectors of the first principal Cauchy stress near the suturing site

\section{Conclusion}

A numerical approach has been presented to simulate the large deformation behaviour of soft tissues. Overall, the model produced reasonable results. The numerical simulation revealed detailed stress and displacement information in the analysed domain that would be potentially useful in studies of soft tissue mechanics. The main features in the results are explainable in terms of the applied attachment forces and resistance due to deformation of the elastic soft tissues.

\section{References}

[1] Larrabee, W. F., Jr., A finite element model of skin deformation-I. Biomechanics of skin and soft tissue: A review. Laryngoscope, April, 399-405, 1986.

[2] Bro-Nielson M. and Cotin S., Real-time volumetric deformable models for surgery simulation using finite elements and condensation. Computer Graphics forum. 15(3), 57-66 (Eurographics Forum'96), 1996.

[3] Ferrant, M., Warfield, S. K., Guttman, C. R. G., Jolesz, F. A. and Kikinis, R., 3D image matching using a finite element based elastic deformation model. MICCAI'99 (eds by Talor C. and Colchester A.). Springer-Verlag. 202-209, 1999.

[4] Nienhuys, H.-W. and van der Stappen A. F., Combining finite element deformation with cutting for surgery simulations. EuroGraphics Short Presentations (de Sousa A. and Torres J. eds.), 43-52, 2000. 
[5] Azar, F. S., Metaxas, D. N. and Schnall, M. D., A deformable finite element model of the breast for predicting mechanical deformations under external perturbations. Academic Radiology, 8 (10), 965-975, 2001.

[6] Picinbono, G. Lombardo, J. C., Delingette, H. and Ayache N., Improving realism of a surgery simulator: Linear anisotropic elasticity, complex interactions and force extrapolation. Rapport de recherche No. 4018. Institut national de recherche en informatique et en automatique, 2000.

[7] Martin Roth, S. H., Gross M. H., Turello S. and Carls F. R., A Bern-Bezier based approach to soft tissue simulation. Eurographics 98. (Ferreira N. and Gobel M., ed.). 17(3), 1998

[8] Fung Y.C., Biomechanics: Mechanical properties of living tissues. Springer, New York, 1993.

[9] Lott-Crumpler D. A. and Chaudhry, Optimal patterns for suturing wounds of complex shapes to foster healing. J. of Biomech. 34, 51-58, 2001

[10] Maurel W., Wu Y., Magnenat Thalmann N. and Thalmann D., Biomechanical models for soft tissue simulation. Springer, 1998.

[11] Hibbitt, H. D., Marcal, P. V. and Rice, J. R., A finite element formulation for problems of large strain and large displacement. Int. J. Solids Struct. 6, 1069.

[12] Kleiber M., 1989. Incremental finite element modelling in non-linear solid mechanics. Chichester. Ellis Horwood, 1970.

[13] Crisfield, M. A., Non-linear finite element analysis of solids and structures. John Wiley \& Sons Ltd, 1991.

[14] Anon, Abaqus Manuals: Theory. Version 5.7, 1997.

[15] Zhou, Z., Theory of three-dimensional consolidation on large strain theory, In the Proceedings of Int. Con. on Engineering problems of regional soils, Beijing, China, 538-540, 1988.

[16] Zhou Z., Large strain consolidation analysis of soft clay foundation, Journal of water resource and navigation (in Chinese). No. 1, 105-110, 1992. 
